\title{
Simulation of the dynamic range extension system for the LHAASO-WCDA experiment
}

\section{Xiurong Li}

Key Laboratory of Particle Astrophysics, Institute of High Energy Physics, CAS, Beijing 100049, China. E-mail: lixr@ihep.ac.cn

\section{Cheng Liu}

Key Laboratory of Particle Astrophysics, Institute of High Energy Physics, CAS, Beijing 100049, China. E-mail: lixr@ihep.ac.cn

\section{Xiaohan Ding}

Key Laboratory of Particle Astrophysics, Institute of High Energy Physics, CAS, Beijing 100049, China. E-mail: lixr@ihep.ac.cn

\section{Wenyan Du}

Key Laboratory of Particle Astrophysics, Institute of High Energy Physics, CAS, Beijing 100049, China. E-mail: lixreihep.ac.cn

\section{Hangrong Wu}

Key Laboratory of Particle Astrophysics, Institute of High Energy Physics, CAS, Beijing 100049, China. E-mail: lixr@ihep.ac.cn

\section{Huicai Li}

Key Laboratory of Particle Astrophysics, Institute of High Energy Physics, CAS, Beijing 100049, China. E-mail: lixr@ihep.ac.cn

The Water Cherenkov Detector Array (WCDA) is one of the major components of the Large High Altitude Air Shower Observatory (LHAASO). The whole WCDA is subdivided into 3120 detector cells, each has $5 \mathrm{~m} \times 5 \mathrm{~m}$ area with an 8 inch PMT residing in the water at the effective depth of 4 $\mathrm{m}$. In order to extend the dynamic range of the detector to the energy more than $100 \mathrm{TeV}$ for the cosmic ray spectrum measurement, 900 cells of the WCDA array are put another 1 inch PMT at side of the big PMT. A fast simulation code based on Geant 4 is developed for purpose of studying the performance of the extension system. In this paper, the fast simulation method is introduced, and CORSIKA and Geant 4 simulation results, such as the shower core and the lateral distribution of the shower signals of high energy cosmic rays are presented.

35th International Cosmic Ray Conference - ICRC2017

10-20 July, 2017

Bexco, Busan, Korea 


\section{Intorduction}

The origin and acceleration of cosmic rays has been a puzzle for a century, which contains lots of mysterious about the universe [1]. Energy spectrum of mixed cosmic rays follows a simple power law with some fine structures, such as the knee at $3^{15} \mathrm{eV}$, the second knee at $4^{17} \mathrm{eV}$, the ankle at $4^{17} \mathrm{eV}$ and unconfirmed GZK cutoff at $6^{19} \mathrm{eV}$ [2]. The fine structures has relationship with the origin and acceleration of cosmic rays, so the accurate measurement of energy spectra of cosmic rays is very important, especially the energy spectra of individual elements. Because of the limitation of space load and the low flux of cosmic rays at high energy, it is difficult to detect the energy of cosmic rays above $100 \mathrm{TeV}$ with space-bored experiments at the top of atmosphere. Presently, cosmic ray above $100 \mathrm{TeV}$ can only be measured by ground based experiments, by detecting the extensive air showers (EAS) produced by the interaction of cosmic particles with nuclei of the Earth's atmosphere. The ground-based measurements detect the information of the EAS secondary particles, and then reconstruct energy of primary particles. The energy of cosmic ray measured on the ground relys on simulation and interaction models, and is also limited by the large fluctuation of EAS. Thus, different experiments get different knee energy, because of the lack of energy scales between different experiments [3].

The Large High Altitude Air Shower Observatory (LHAASO) is designed to search for cosmic ray sources by the measurement of $\gamma$ rays and to study cosmic ray physics from tens of $\mathrm{TeV}$ to a few hundreds of PeV [4]. LHAASO will accurately measure the energy spectra of cosmic rays for single elements and improve the chaotic situation in measurement of energy spectra, with three major detector arrays: LHAASO-WCDA, LHAASO-KM2A, LHAASO-WFCTA.

The Water Cherenkov Detector Array (WCDA), which is one of the main components of LHAASO, functions as surveying the northern sky for high-energy $\gamma$ ray sources at the energy range from $100 \mathrm{GeV}$ to $30 \mathrm{TeV}$. The WCDA has an area of 78,000 $\mathrm{m}^{2}$, and is sub-divided into 3,120 cells by black curtains, each has $5 \mathrm{~m} \times 5 \mathrm{~m}$ area with an 8 inch PMT (big PMT) residing in the water at the effective depth of $4 \mathrm{~m}$. In order to extend the dynamic range of the detector to the energy more than $100 \mathrm{TeV}$ for the cosmic ray spectrum measurement, 900 cells of the WCDA array are put another 1 inch PMT (small PMT) at side of the big PMT. The new system of WCDA with small PMTs is called the dynamic range extension system for the LHAASO-WCDA, and in short as WCDA++. The WCDA++ system will work together with WFCTA and KM2A to measure the energy spectra of cosmic rays with energy from $100 \mathrm{TeV}$ to $10 \mathrm{PeV}$, which can give good energy scaler between space-bored experiments and ground-based measurements. The WCDA++ system detects the Cherenkov light which produced in water by cascade process of secondary particles that falling into the water cell. The number of photon electron (NPE) of PMTs reflects the density and energy of secondary particles, which is able to reconstruct the core position and is sensitive to the identity of primary particles.

The simulation of LHAASO is divide to the air shower part and the detector part. The air showers initiated by cosmic rays is simulated with CORSIKA program and the information of secondary particles reaching the WCDA is gathered. Then the response of the secondary particles in the WCDA water pool is simulated with Geant4 program. A fast simulation code based on Geant4 is developed for purpose of studying the performance of the WCDA++ system with high energy cosmic rays, and also for the reconstruction and data analysis. In this talk, the fast simulation 
method is introduced, and CORSIKA and Geant 4 simulation results, such as the signal at shower core and the lateral distribution of the shower signals for high energy cosmic rays are presented.

\section{The EAS study with CORSIKA simulation}

The high energy cosmic rays interact with the helium in the atmosphere, which can generate huge numbers of particles in the LHAASO site. We simulated proton and iron EAS events with CORSIKA program, and get the information of secondary particles. The secondary particles spread at hundreds of meters from the core at the altitude of LHAASO site, with most around the core area. Most secondary particles are gamma, electons, muons and hadrons. The energy of secondary particle has a wide distribution which can reach $\mathrm{TeV}$ level when the energy of primary particle is $10 \mathrm{PeV}$. Huge number of secondary particles can hit each WCDA $5 \times 5 \mathrm{~m}$ cell, especially the core cell. Charged particles deflected by galactic magnetic field, and the core position can have an offset in the east-west direction. Iron has more nucleons and charge, which means more chance to have interaction with the helium in the atmosphere, and bigger core offset caused by the magnetic field. The left of Fig. 1 is the y position distribution of secondary particles caused by $100 \mathrm{TeV}$ proton and iron cosmic ray with $\mathrm{x}$ within $\pm 2.5 \mathrm{~m}$, while $\mathrm{x}$ here in the CORSIKA program points to the north and y points to the west. The right of Fig. 1 is the number of secondary particles in each WCDA cell caused by a $100 \mathrm{TeV}$ proton and a $100 \mathrm{TeV}$ iron. A $100 \mathrm{TeV}$ proton has about 8500 secondary particles in the WCDA core cell, while a $100 \mathrm{TeV}$ iron has about 400 secondary particles in the core cell. The number of secondary particles caused by proton EAS is about 20 times of the number from iron with the same energy. The core offset of iron is much bigger than proton, and the lateral distribution of secondary particles from iron is flatter than and proton. So the WCDA++ can be a good system to detect the core position of cosmic rays and to do particle identification of different species of cosmic rays.
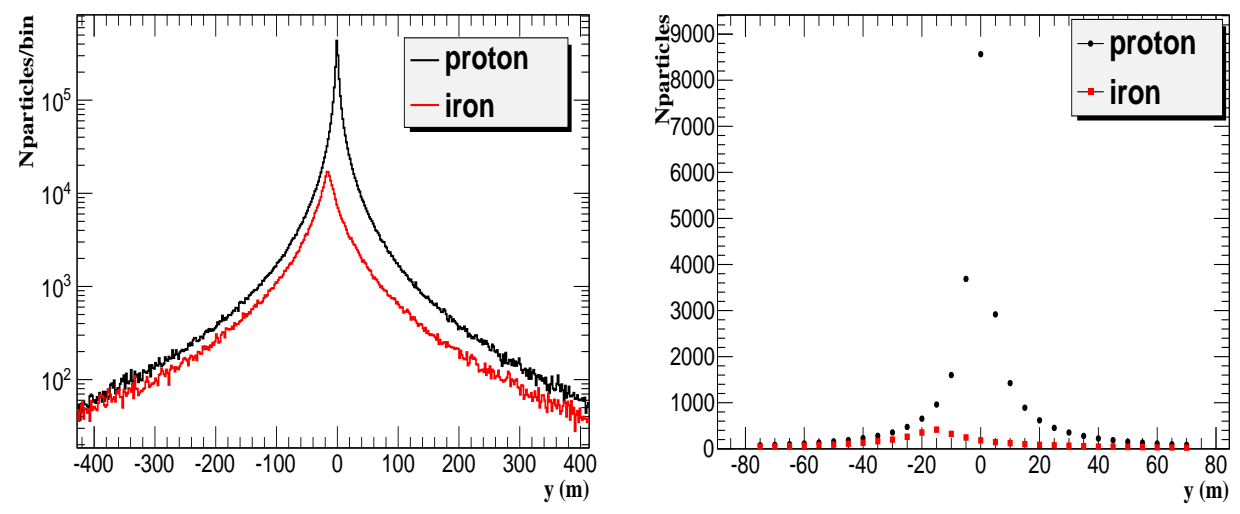

Figure 1: The left is the y position distribution of secondary particles caused by $100 \mathrm{TeV}$ proton and iron EAS with $\mathrm{x}$ within $\pm 2.5 \mathrm{~m}$; The right is the number of secondary particles in each WCDA cell caused by a $100 \mathrm{TeV}$ proton and a $100 \mathrm{TeV}$ iron. 


\section{Geant4 fast simulation}

The Geant4 program is based and modified from the WCDA Geant4 program [5]. In order to save CPU and the memory, we reserve and transmit the NPE numbers of each PMT, instead of reserve the total information of photons hitting the PMT. The detector setting is based on the LHAASO-WCDA design, and the water quality setting is based on the prototype detector at Yangbajing[6]. We add one 1 inch PMT in each of 900 cells at $30 \mathrm{~cm}$ left of the big PMT at the height of big PMTS to do simultion. The left of Fig. 2 is the simulated average NPE of $100 \mathrm{MeV}$ $\gamma$ generated form different distance form the center of big PMT at the water surface, and right of Fig. 2 is the averaged NPE of $2 \mathrm{GeV}$ muon.
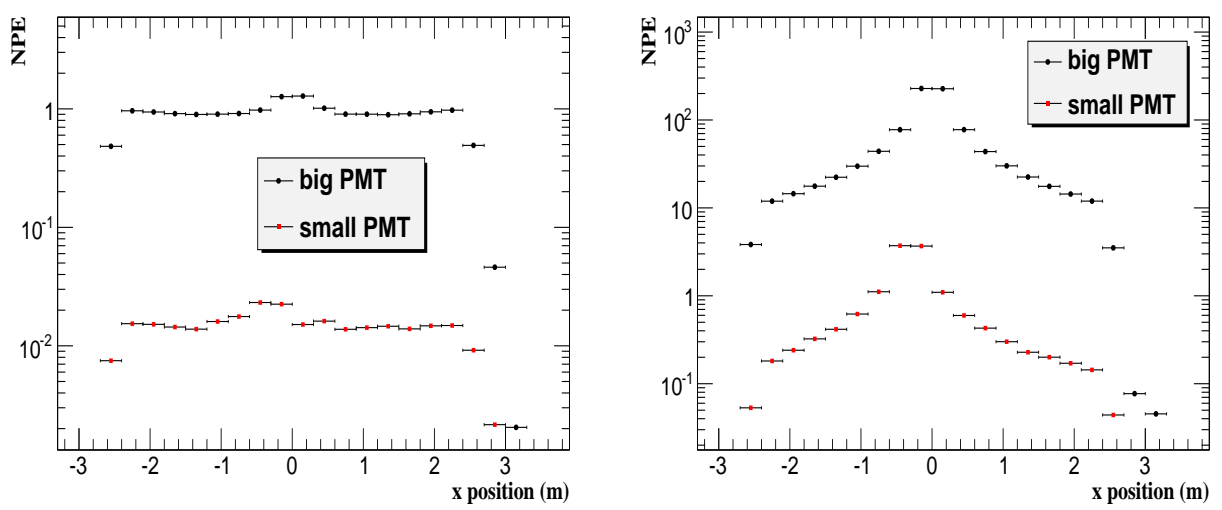

Figure 2: The left is the simulated average NPE of $100 \mathrm{MeV}$ gamma generated form different distance form the center of big PMT at the water surface, and right is the NPE of $2 \mathrm{GeV}$ muon.

We tried different methods to save the Geant 4 running time, for example, thinning the Cherenkov photon numbers, killing photons depending on PMT collection efficiency beforehand, and using the method of parameterization for electronic particles. A charged particle with high speed can generate about 300 Cherenkov photons in the water per centimeter. Thinning photons with a factor of 20 means simulating 1/20 of the generated photons and using 20 times of the NPE result simulated to get the last simulation result, which can make the running time to be 1/16 and give similar simulation result compared with no thinning for single particles as well for EAS showes. Thnning photons with a factor of 300 can give good result of mean value of NPE, but can enlarge the error when the NPE numbers is not large. Killing photons depending on PMT collection efficiency beforehand can save at least half of running time. Another method to save CPU running time is the parameterization method, which means doing the simulation of electronic particles with different energy and at each positon area and each injection theta angle bin and phi angle bin beforehand and reserve the NPE values respectively. Then the reserved NPE values can be used when the Geant 4 simulation of EAS secondary particles is done, according to the energy and position as well as angle information of the secondary particles. The left of Fig. 3 is the NPE values of $\gamma$ at different energy with different method of simulation, and the green point is the reserved NPE parameters. We get similar averaged NPE result for single $\gamma$ with the method of Geant 4 full simulation, simulation with thinning method and simulation with parameterization method. The right of Fig. 3 is the time consuming to simulate a $\gamma$ with different method. The time of full simulation and simulation 
with thinning method increase with the particle energy, while the time of parameterization method keeps at about 0.002 s for each particle.
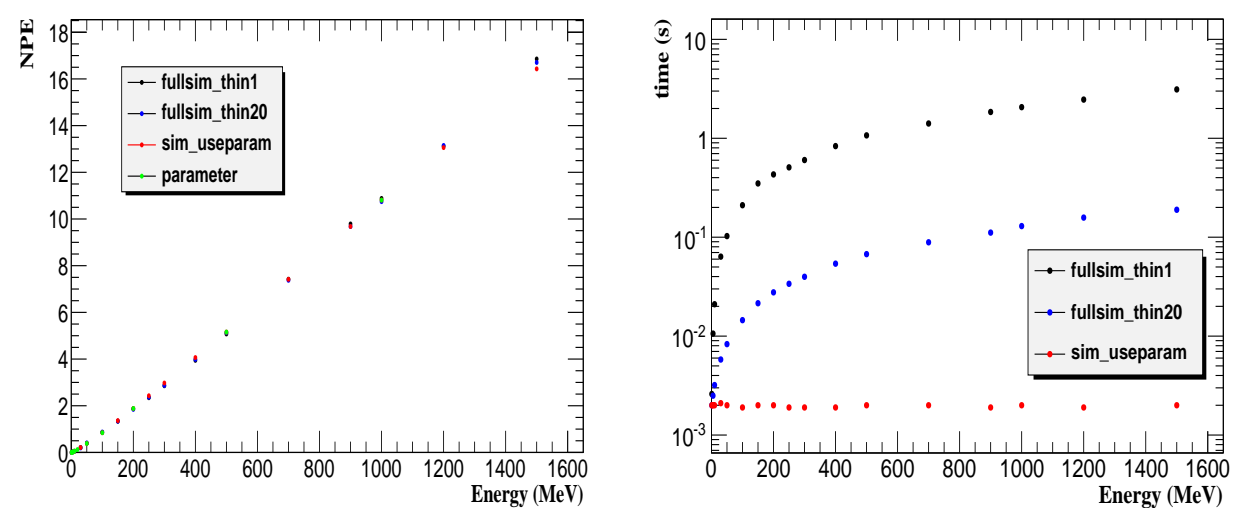

Figure 3: The left is the NPE values of $\gamma$ at different energy with different method of simulation; The right is the time consuming to simulate a $\gamma$ with different method.

\section{Geant4 simulation result of cosmic showers}

The WCDA Geant4 running time with the thinning method is less than 1 hours for a $100 \mathrm{TeV}$ proton EAS. We generated 100 vertical showers of $100 \mathrm{TeV}$ proton and $100 \mathrm{TeV}$ iron, and simulated to get NPE of different PMTs of WCDA++. The left of Fig. 4 is the mean NPE of big and small PMTs caused by a $100 \mathrm{TeV}$ proton and $100 \mathrm{TeV}$ iron respectively. The NPE of big PMT at core cell get about $4 \times 10^{4}$ for a $100 \mathrm{TeV}$ proton while the value for a $100 \mathrm{TeV}$ iron is about $4 \times 10^{2}$, and the lateral distribution of NPE from proton is much steeper than that of iron. We simulated about 5000 proton EAS and 5000 iron EAS with about 30 degree of polar angle from $100 \mathrm{TeV}$ to $1 \mathrm{PeV}$, and get the Geant 4 simulation result. The right of Fig. 4 is the NPE of the small PMT at the core cell for different energy of proton and iron EAS, while the black points are for iron and red points are for iron. Normally the NPE caused by proton EAS is bigger than that from iron at the core cell, with big fluctuation.

\section{Summary}

The Geant4 fast simulation for WCDA++ is developed and many methods to speed up the program is tried, which can still be optimized. The simulation results such as the signals at shower core and the lateral distribution of the signals for proton and iron EAS are studied. Then we will do simulation of different species of cosmic showers at different energy to develop the method of shower core reconstruction and particle identification.

This work is supported by National Natural Science Foundation (NSFC) of China under contacts (No. 11635011, NO.11375210,No.11375224, No.11405181, No.11475190), the Chinese Academy of Science, Institute of High Energy Physics, the Key Laboratory of Particle Astrophysics, CAS. 

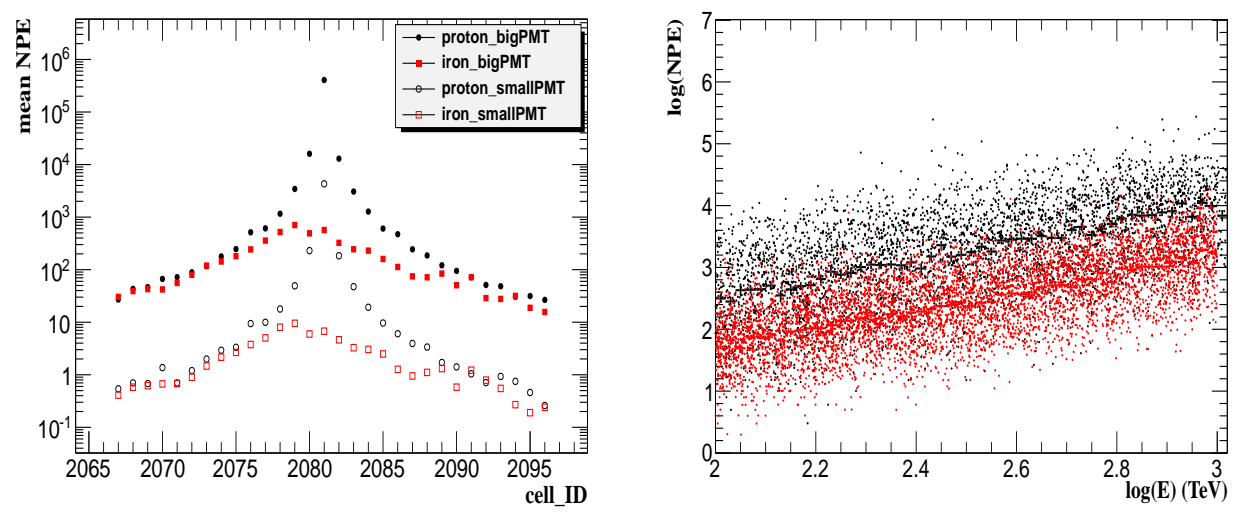

Figure 4: The left is the mean NPE of big and small PMTs caused by a $100 \mathrm{TeV}$ proton and $100 \mathrm{TeV}$ iron vertical EAS respectively; The right is the NPE of the small PMT at the core cell for different energy of proton and iron EAS with about 30 degree of polar angle.

\section{References}

[1] E.G. Berezhko,Astrophys.J. 661,L175(2007).

[2] K.Greisen, Phys.Rev.Lett., 1966, 16.

[3] V.Berezinsky,J. Phys.Conf. Ser.,2008, 120(1): 012001.

[4] Z.Cao (for LHAASO Coll.), Chin.Phys.C34 (2010) 249-252.

[5] H.R. Wu, Proceeding of Science, ICRC 2017.

[6] Hui-Cai Li,Zhi-Guo Yao,Chun-Xu Yu, et al. Chinese Physics C, 2017, 41(2): 26002-026002. 